\title{
Cyclic Mechanical Stretching Induces Autophagic Cell Death in Tenofibroblasts Through Activation of Prostaglandin E2 Production
}

\author{
Hua Chen ${ }^{\mathrm{a}}$ Liyang Chen $^{\mathrm{b}}$ Biao Cheng ${ }^{\mathrm{b}}$ Chaoyin Jiang ${ }^{\mathrm{a}}$ \\ aDepartment of Orthopedics, Shanghai Jiao Tong University Affiliated Sixth People's Hospital, Shanghai, \\ bDepartment of Orthopedics, Shanghai Tenth People's Hospital, Tongji University, School of Medicine, \\ Shanghai, China
}

\section{Key Words}

Prostaglandin E2 • Autophagy • Cell death • Tendinopathy

\begin{abstract}
Background/Aims: Autophagic cell death has recently been implicated in the pathophysiology of tendinopathy. Prostaglandin E2 (PGE2), a known inflammatory mediator of tendinitis, inhibits tenofibroblast proliferation in vitro; however, the underlying mechanism is unclear. The present study investigated the relationship between PGE2 production and autophagic cell death in mechanically loaded human patellar tendon fibroblasts (HPTFs) in vitro. Methods: Cultured HPTFs were subjected to exogenous PGE2 treatment or repetitive cyclic mechanical stretching. Cell death was determined by flow cytometry with acridine orange/ethidium bromide staining. Induction of autophagy was assessed by autophagy markers including the formation of autophagosomes and autolysosomes (by electron microscopy, AO staining, and formation of GPF-LC3-labeled vacuoles) and the expression of LC3-II and BECN1 (by western blot). Stretching-induced PGE2 release was determined by ELISA. Results: Exogenous PGE2 significantly induced cell death and autophagy in HPTFs in a dose-dependent manner. Blocking autophagy using inhibitors 3-methyladenine and chloroquine, or small interfering RNAs against autophagy genes Becn-1 and Atg-5 prevented PGE2-induced cell death. Cyclic mechanical stretching at $8 \%$ and $12 \%$ magnitudes for $24 \mathrm{~h}$ significantly stimulated PGE2 release by HPTFs in a magnitude-dependent manner. In addition, mechanical stretching induced autophagy and cell death. Blocking PGE2 production using COX inhibitors indomethacin and celecoxib significantly reduced stretching-induced autophagy and cell death. Conclusion: Taken together, cyclic mechanical stretching induces autophagic cell death in tenofibroblasts through activation of PGE2 production.
\end{abstract}

H. Chen and L. Chen contributed equally to this work and should be considered co-first authors.

Chaoyin Jiang,

and Biao Cheng,

KARGER 125
Department of Orthopedics, Shanghai Jiao Tong University Affiliated Sixth People's Hospital, Shanghai 200233, (China) and Department of Orthopedics, Shanghai Tenth People's Hospital, Tongji University, School of Medicine, Shanghai (China)

E-Mail doctor_jiangcy@126.com and E-Mail cbiao6@126.com 


\section{Cellular Physiology Cell Physiol Biochem 2015;36:24-33 \\ \begin{tabular}{l|l|l} 
DOI: 10.1159/000374050 & (C) 2015 S. Karger AG, Basel \\
and Biochemistry
\end{tabular} \\ Chen et al.: Cyclic Mechanical Stretching Triggers Autophagic Cell Death via $\mathrm{PGE}_{2}$}

\section{Introduction}

Tendinopathy is a common clinical problem in sports and occupational settings in modern society [1]. Chronic or acute tensile overload can lead to tendon inflammation and degeneration. Prostaglandin E2 (PGE2) is a key inflammatory mediator in tendinopathy. When the rotator cuff is torn, interleukin-1 $\beta$ (IL-1 $\beta$ ) is produced in the torn tendon, stimulating cyclooxygenase 2 (COX-2) expression and subsequent prostaglandin E2 (PGE2) production [2]. In in vitro model systems, mechanical loading often don fibroblasts or isolated tendon increases PGE2 production and release [3-5]. Exogenous PGE2 treatment inhibits tendon fibroblast proliferation and collagen synthesis in vitro [6] and causes localized tendon disorganization and degeneration in vivo $[7,8]$. However, the mechanism by which PGE2 inhibits tendon fibroblast proliferation is unclear.

Excessive cell death in degenerative tendon can impair collagen synthesis and repair, contributing to the pathophysiology of tendinopathy [9]. Apoptosis and autophagy are both forms of programmed cell death. Apoptosis is a recognized causative factor in tendinopathy. Increased apoptosis has been described in human tendinopathic tissues $[10,11]$ as well as in animal tendon after high-strain mechanical loading [12]. In in vitro systems, mechanical stretching can induce apoptosis in human tendon fibroblasts via oxidative stress-associated pathways [13]. Recently, autophagy has been implicated as another contributing factor in tendinopathy. Autophagy, a basic catabolic mechanism to remove unnecessary or dysfunctional cellular components through the actions of lysosomes, promotes cellular survival during starvation by maintaining cellular energy levels [14]. However, autophagy can cause degradation of proteins and organelles essential for cell survival, which eventually leads to cell death [15]. Both apoptosis and autophagic cell death have been observed in human tendinopathic tissues $[16,17]$, with up to $51.9 \%$ autophagic cell death being detected in tendon samples collected from patients undergoing surgery for rotator cuff tears [17]. However, the factors causing autophagic cell death in tendinopathic tissues are not clear. In the present study, we investigated the effects of exogenous PGE2 and repetitive mechanical loading on the induction of autophagy and cell death in human patellartendon fibroblasts in vitro. The relationship between mechanical loading, PGE2 production, and autophagic cell death were investigated.

\section{Materials and Methods}

\section{Reagents}

A Acridine orange (AO), ethidium bromide (EB), 3-MA, chloroquine, were purchased from SigmaAldrich Canada (Oakville, ON, Canada). Hoechst 33342 staining kit was purchased from Molecular Probes (Eugene, OR). All the antibodies used in this study were bought from Cell Signaling Technology, Inc. (Beverly, MA, USA).

Isolation and culture of humanpatellar tendon fibroblasts

Human patellar tendon fibroblasts (HPTFs) were isolated from tendon pieces trimmed from patellar tendon autografts of two healthy male donors ( 16 and 31 years old) and one healthy female donors (25 years old ) who underwent reconstruction of the anterior cruciate ligament. The protocol for obtaining the tendon samples was approved by the Research Ethics Committee of Shanghai Sixth People's Hospital (Shanghai, China). HPTFs were isolated and expanded as described previously [4]. Briefly, the tendon samples were thoroughly washed with phosphate buffered saline (PBS), minced, and incubated in a 100-mm Petri dish in DMEM containing 10\% heat-inactivated fetal bovine serum (FBS, Invitrogen, Carlsbad, CA, USA) and 1\% penicillin/streptomycin (P/S; Invitrogen) at $37^{\circ} \mathrm{C}, 5 \% \mathrm{CO}_{2}$ in a humidified incubator. When the cells grew to $100 \%$ confluence, they were harvested and subcultured. Cells after 5 to 7 passages were used in stretching experiments. 


\section{Cellular Physiology Cell Physiol Biochem 2015;36:24-33 \begin{tabular}{l|l|} 
DOI: 10.1159/000374050 2015 & O 2015 S. Karger AG, Basel \\
\hline
\end{tabular} and Biochemistry \\ Chen et al.: Cyclic Mechanical Stretching Triggers Autophagic Cell Death via $\mathrm{PGE}_{2}$}

Cell death determination

Cell death was determined by flow cytometry (BD FACS Calibur) using acridine orange/ethidium bromide (AO/EB) staining. Live cells are permeable to AO but not EB and stained green. Dead cells are permeable to both AO and EB and stained orange. Data were acquired and analyzed using CellQuest software (Becton Dickinson, USA). In addition, cell membrane permeability was assessed microscopically using both AO/EB and Hoechst staining.

Quantification of Acidic Vesicular Organelles (AVO) with AO Staining

Autophagy is the process of sorting cytoplasmic constituents into vesicles known as AVOs (autophagosomes and autolysosomes) for lysosomal degradation. To assess the induction of autophagy, we measured the formation of AVOs using flow cytometry with AO staining as described previously [18, 19]. AO is a fluorescent weak base that accumulates in acidic spaces. AVOs fluoresce bright red in AO-stained cells [19]. Briefly, cells were collected by centrifugation, resuspended in $1 \mathrm{ml}$ PBS, and stained with AO (100 $\mu \mathrm{g} /$ $\mathrm{ml}$ ) for 15 - $20 \mathrm{~min}$. After washing twice with PBS, cells were resuspended in $0.3 \mathrm{ml}$ PBS and analyzed on a flow cytometer (BD FACS Calibur) using CellQuest software.

\section{Detection of autophagosomes with GFP-LC3 labeling}

GFP-LC3-expressing constructs were obtained from Addgene (Cambridge, MA, USA). Cells were transfected with $1 \mu \mathrm{g}$ of GFP-LC3 cDNA in a mammalian expression vector using Lipofectamine 2000 reagent (Invitrogen) for $4 \mathrm{~h}$. The transfected cells were seeded in six-well plates ( $2 \times 10^{5}$ cells/well) and cultured overnight. On the next day, cells were treated with PGE2 at $37^{\circ} \mathrm{C}$ for $24 \mathrm{~h}$, and GFP-LC3-labeled vacuoles (autophagosomes) were counted as described previously [18] under an Olympus microscope equipped with a coolsnap camera. When $3-\mathrm{MA}(2 \mathrm{mM})$ or chloroquine $(30 \mu \mathrm{M})$ was included in the treatment, cells were pre-incubated with the compound at $37^{\circ} \mathrm{C}$ for $1 \mathrm{~h}$ prior to PGE2 treatment.

\section{Silencing of Becn-1 and ATG5 by siRNAs}

The small interfering RNAs (siRNA) specific for human Becn-1 and ATG5 were purchased from Dharmacon (Lafayette, CO, USA). A scrambled RNA from was used as the control. The same amount of cells were seeded in a Petri plate $(100 \times 20 \mathrm{~mm})$ and incubated at $37^{\circ} \mathrm{C}$ overnight. On the next day, cells were transfected with siRNA (scrambled, Becn-1, or ATG5) for 6 h using Lipofectamine RNAImax (Invitrogen) following manufacturer's instructions. The transfected cells were collected, seeded in six-well plates $(2 \mathrm{x}$ $10^{5}$ cells/well), and cultured overnight. On the next day, cells were treated with PGE2 at $37^{\circ} \mathrm{C}$ for $24 \mathrm{~h}$. Cell death and the formation of AVOs were analyzed as above. To assess the formation of autophagosomes, cells were transfected with GFP-LC3 prior to siRNA transfection.

\section{Ultrastructural analysis by electron microscopy}

Cells were fixed in 2\% paraformaldehyde for $2 \mathrm{~h}$ and then post-fixed in 1\% 0s04 for $1.5 \mathrm{~h}$. After washing with PBS, the cells were stained with 3\% aqueous uranyl acetate for $1 \mathrm{~h}$, washed with PBS, dehydrated with graded alcohol, and embedded in Epon-Araldite resin (Canemco). Ultrathin sections were cut on a Reichert ultramicrotome, counterstained with $0.3 \%$ lead citrate, and examined on a Philips EM420 electron microscope.

\section{Western blot analysis}

Cells were lyzed in NP-40 lysis buffer (50 mM HEPES, $150 \mathrm{mM} \mathrm{NaCl}, 50 \mu \mathrm{M} \mathrm{ZnCl}, 50 \mu \mathrm{M} \mathrm{NaF}, 2 \mathrm{mM}$ EDTA, $1 \mathrm{mM}$ sodium vanadate, 1.0\% NP-40, $2 \mathrm{mM}$ phenylmethylsulfonyl fluoride; $\mathrm{pH} 7.25$ ). The protein concentration was determined using the Bradford assay. Proteins were separated by sodium dodecyl sulfate-polyacrylamide gel electrophoresis (SDS-PAGE) and transferred to nitrocellulose membranes (Millipore, USA). After blocking with 5\% non-fat dry milk for $1 \mathrm{~h}$, the membranes were incubated with antiLC3-I, anti-LC3-II, anti-BECN1, anti-Atg5, and anti- $\beta$-actin antibodies, respectively, at $4{ }^{\circ} \mathrm{C}$ overnight. After washing, the membranes were incubated with secondary antibodies conjugated to horseradish peroxidase. Protein bands were visualized using enhanced chemiluminescence reagents (Pierce (Thermo Scientific), Waltham, USA). Tris-Tricine SDS-PAGE was used for the detection of LC3-I and LC3-II. Tris-glycine SDSPAGE was used for the detection of all other proteins. 


\section{Cellular Physiology Cell Physiol Biochem 2015;36:24-33

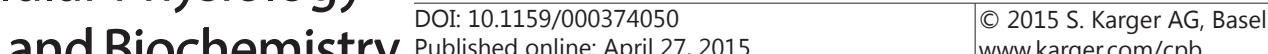 \\ Chen et al.: Cyclic Mechanical Stretching Triggers Autophagic Cell Death via PGE ${ }_{2}$}

In vitro cell stretching experiments

Cyclic mechanical stretching was applied to cultured HPTFs in an in vitro model system as described previously [4, 20,21]. Briefly, A total of $2 \times 10^{5} \mathrm{HPTF}$ were grown on the microgrooved surface of a custommade silicone dish in DMEM containing 10\% FBS and 1\% P/S. The silicon dish was pre-coated with $10 \mu \mathrm{g} /$ $\mathrm{ml}$ of ProNectin-F (Sigma, USA) to promote cell attachment. After $24 \mathrm{~h}$, the medium was replaced with fresh DMEM containing 1\% FBS and 1\% P/S. After another 36 48 h incubation, the cells were cyclically stretched with $4 \%, 8 \%$, or $12 \%$ magnitude at a constant stretching frequency of $0.5,1$, or $1.5 \mathrm{~Hz}$ for a duration of 24 h. Non-stretched HPTFs were used as the control. After a $4 \mathrm{~h}$ rest, the concentration of PGE2 in the culture media was determined by ELISA. Cell death and the formation of AVOs were analyzed as described above. To assess the formation of autophagosomes, cells transfected with GFP-LC3 were used in the stretching experiments. When indomethacin $(25 \mu \mathrm{M})$ or celecoxib $(40 \mu \mathrm{M})$ was included in the treatment, cells were pre-incubated with the compound for $1 \mathrm{~h}$ prior to mechanical stretching.

$P G E 2$ release by ELISA

The PGE2 concentration in the culture mediawas determined using a PGE2 ELISA kit from R\&D Systems Inc. (USA) following manufacturer's instructions. The absorbance at $405 \mathrm{~nm}$ was measured on a Spectra MAX190 microplate reader (Molecular Devices, USA). The PGE2 concentration was calculated using a standard curve created with PGE2 solutions of known concentrations.

\section{Statistical analysis}

All data are expressed as means \pm s.e. (standard error). All experiments were performed at least three times. Each experiment was performed in two or more replicates. Data were analyzed using Excel and SigmaPlot. Results from different groups were compared using Student's $t$-test. Differences with a P value less than 0.05 were considered statistically significant.

\section{Results}

PGE2 induces cell death in tenofibroblasts

We isolated and expanded HPTFs as described previously [4]. Sufficient amounts of cells were obtained after 5 passages for experimental studies. To test the effects of PGE2 on tenofibroblast viability, HPTFs were treated with exogenous PGE2 at increasing concentrations (range: $1-15 \mathrm{ng} / \mathrm{ml}$ ) for a duration of 12, 24, or $48 \mathrm{~h}$. Immediately after treatment, cells were stained with either AO/EB or Hoechst 33342 and examined under a fluorescence microscope. PGE2 exposure increased the number of cells stained positive for EB (Fig. 1A) and Hoechst (Fig. 1B) dyes, both indicating increased plasma membrane permeability. Flow cytometric analysis of AO/EB-stained cells showed that exogenous PGE2 induced HPTF cell death in a dose and time-dependent manner (Fig. 1C). Significant induction of cell death was observed at all PGE2 concentrations except $1 \mathrm{ng} / \mathrm{ml}$, the lowest concentration used in the study.

\section{PGE2 induces autophagy in tenofibroblasts}

Autophagy is characterized by the formation of AVOs (autophagosomes and autolysosomes). To find out whether exogenous PGE2 induces autophagy in tenofibroblasts, we treated HPTFs with $10 \mathrm{ng} / \mathrm{ml}$ PGE2 for $24 \mathrm{~h}$, and examined the morphological changes in subcellular organelles by electron microscopy. AVOs were detected in PGE2-treated but not control cells (Fig. 2A). To quantify the effects of PGE2 on AVO formation, cells were treated with $0-15 \mathrm{ng} / \mathrm{ml}$ PGE2 for $24 \mathrm{~h}$, immediately stained with the AVO marker AO, and subjected to flow cytometric analysis. AVOs fluoresce bright red in AO-stained cells. As shown in Fig. 2B, the percentage of cells stained positive for AVOs increased with the PGE2 concentration. Compared with untreated cells, cells treated with 5, 10 or $15 \mathrm{ng} / \mathrm{ml} \mathrm{PGE2} \mathrm{had}$ significantly greater AVO contents. LC3 is a widely used protein marker for autophagosomes. To directly visualize the formation of autophagosomes, cells were transfected with GFP-LC3 and subjected to PGE2 treatment. Using fluorescence imaging, we detected GFP-LC3-labled 
Fig. 1. PGE2 induces cell death in tenofibroblasts. HPTFs were treated with $1,5,10$ or 15 $\mathrm{ng} / \mathrm{ml}$ PGE2 for a duration of 12,24 or $48 \mathrm{~h}$. Untreated cells were used as the control. Plasma membrane permeability was assessed by $\mathrm{AO} / \mathrm{EB}$ and nuclear fragmentation or condensation was measured via Hoechst 33342 staining. (A) Fluorescent microscopic images of $\mathrm{AO} / \mathrm{EB}$ staining. (B) Fluorescent microscopic images of Hoechst 33342 staining. The white arrows show fragmented or condensed nuclei. (C) Cell death determined by flow cytometric analysis with $\mathrm{AO} / \mathrm{EB}$ staining. $\mathrm{n}=3$, $* \mathrm{P}<0.05$ vs. control.

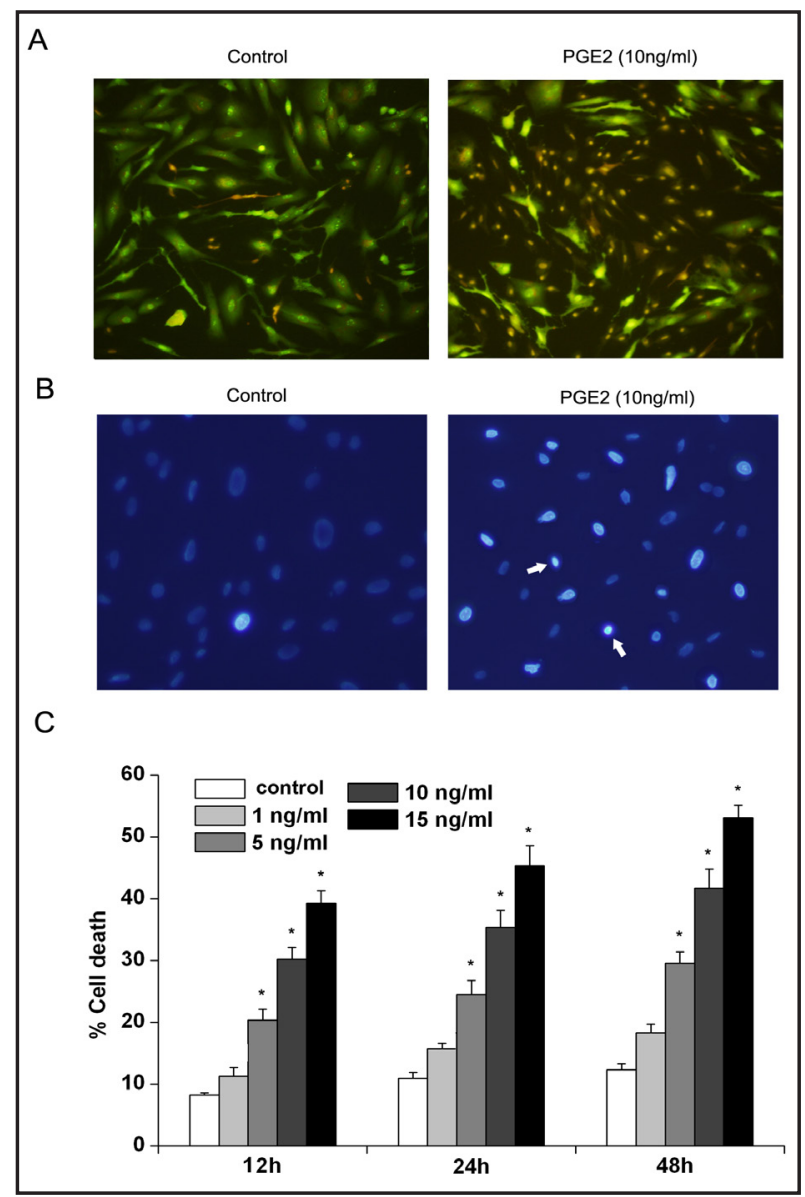

double-membrane vacuoles (autophagosomes) in PGE2-treated but not control cells (Fig. 2C). Moreover, the percentage of cells with GFP-LC3-labled vacuoles increased with the PGE2 concentration (Fig. 2C, bar graph). These data were consistent with results from flow cytometric detection of AVOs using AO staining. To further assess the induction of autophagy, we determined the conversion of LC3-I to LC3-II and the protein level of BECN1 using western blot analysis. The conversion of soluble LC3-I to lipid bound LC3-II is associated with the formation of autophagosomes in autophagy. BECN1 plays an essential role in autophagy initiation. Both are well-recognized protein markers for autophagy. After $24 \mathrm{~h}$ PGE2 treatment, LC3-I stayed essentially unchanged while LC3-II showed a dose-dependent increase (Fig. 2D). Similar to LC3-II, BECN1 was induced by PGE2 in a dose-dependent manner (Fig. 2D). These changes in autophagy protein markers provide further evidence that PGE2 induces autophagy in tenofibroblasts.

Blocking autophagy prevents PGE2-induced cell death in tenofibroblasts

Having demonstrated that PGE2 induces both cell death and autophagy in tenofibroblasts, we investigated whether autophagy contributes to PGE2-induced cell death. Autophagy protein 5 (Atg-5) is an E3 ubiquitin ligase required for autophagosome elongation. Silencing of Becn-1 or Atg- 5 by siRNA significantly decreased the percentage of cells with AVOs or GFPLC3-labled vacuoles after PGE2 treatment (Fig. 3A). Intriguingly, Becn-1 or Atg-5 silencing also significantly attenuated PGE2-induced cell death (Fig. 3A). Similarly, blocking autophagy with autophagy inhibitors 3-methyladenine (3-MA) or chloroquine significantly reduced PGE2-induced cell death (Fig. 3B). Collectively, these results demonstrate that PGE2-induced cell death in tenofibroblastsis mediated by autophagy. 


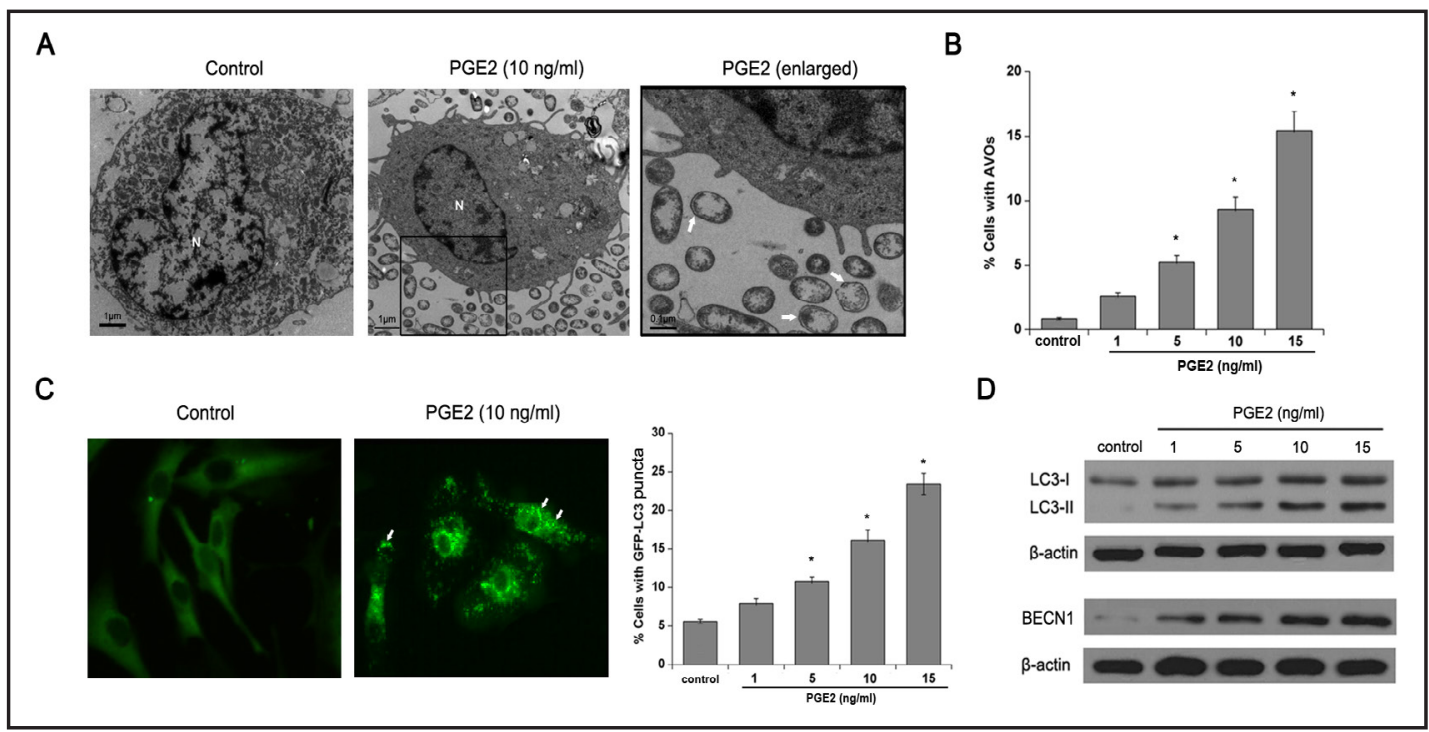

Fig. 2. PGE2 induces autophagy in tenofibroblasts. HPTFs were treated with 1, 5, 10 or $15 \mathrm{ng} / \mathrm{ml}$ PGE2 for 24h. Untreated cells were used as the control. (A) Electron microscopic images. Arrows represent double-membrane vacuoles in PGE2-treated cells (enlarged image). $\mathrm{N}$ represents the nucleus. (B) The percentage of cellswith AVOs determined by flow cytometry. $n=3,{ }^{*} \mathrm{P}<0.05$ vs. control. (C) Representative fluorescent microscopic images showing GFP-LC3-labeled vacuoles (puncta) (left) and the percentage of cells with GFP-LC3 puncta (right). Cells were transfected with GFP-LC3 prior to PGE2 treatment. $\mathrm{n}=3$, ${ }^{*} \mathrm{P}<0.05$ vs. control. (D) The conversion of LC3-I to LC3-II and the expression of BECN1 were determined by western blot. $\beta$-Actin was used as loading control.

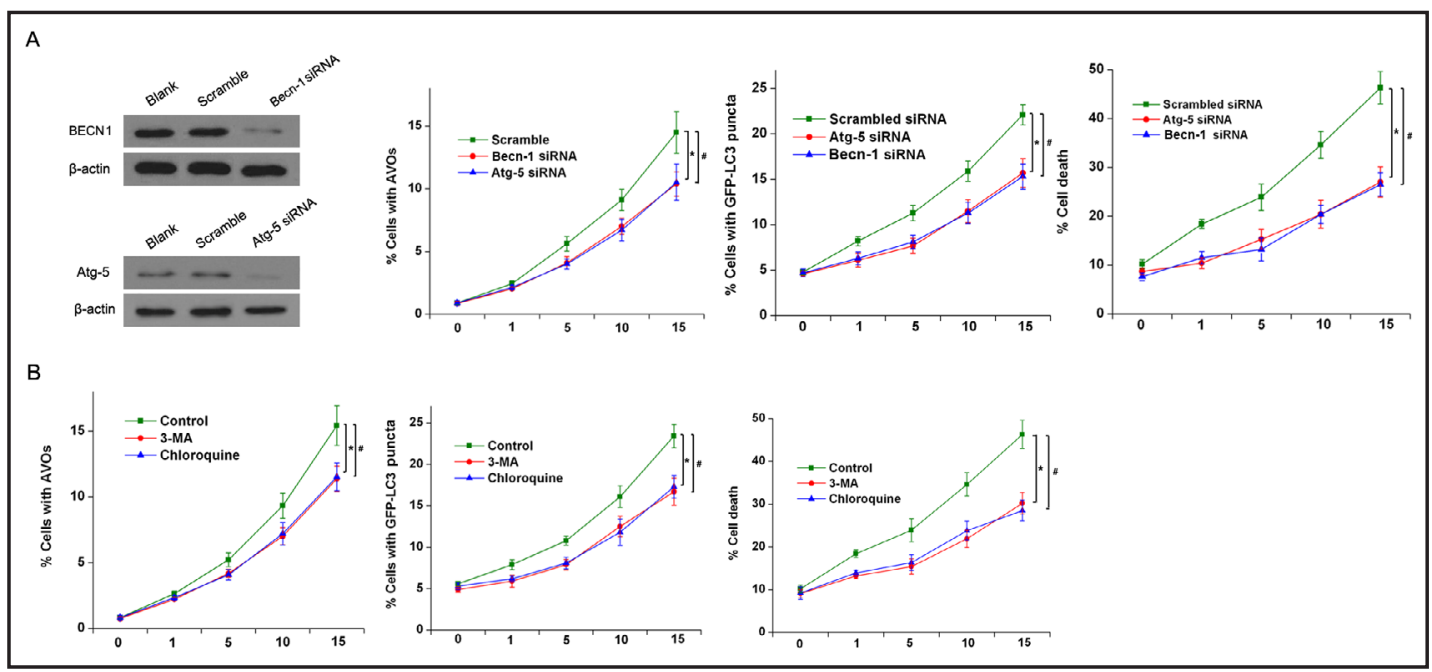

Fig. 3. Blocking autophagy prevents PGE2-induced cell death in tenofibroblasts. HPTFs were subjected to $24 \mathrm{~h}$ treatment with $0-15 \mathrm{ng} / \mathrm{ml}$ PGE2 for $24 \mathrm{~h}$. Autophagy (the formation of AVOs and GFP-LC3-labeled vacuoles) and cell death were assessed. (A) Cells were transfected with scrambled siRNA, Becn-1 siRNA, or Atg-5 siRNA prior to PGE2 treatment. Gene silencing was confirmed by western blot. $\mathrm{n}=3$, , \# $\mathrm{P}<0.05$ vs. scramble. (B) Cells were pre-incubated with 3-MA (2 mM), chloroquine $(30 \mu \mathrm{M})$, or vehicle only (control) prior to PGE2 treatment. $\mathrm{n}=3$, ${ }^{*}$ " $\mathrm{P}<0.05$ vs. control.

Cyclic mechanical stretching induces autophagic cell death in tenofibroblasts through activation of PGE2 production

Previous studies have shown that repetitive mechanical loading stimulates PGE2 production in tenofibroblasts $[3,4]$. Since we had found that exogenous PGE2 induces 


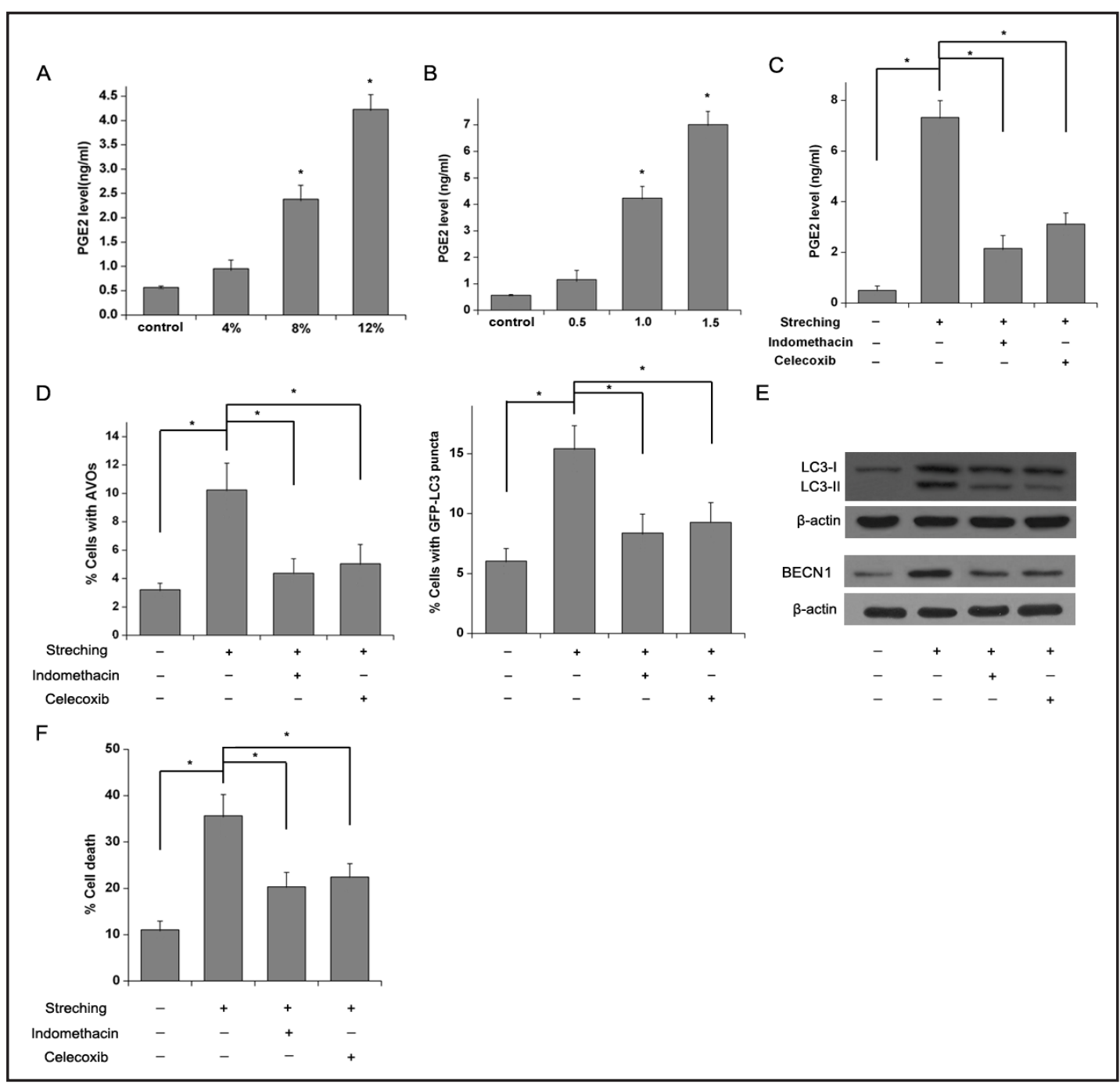

Fig. 4. Cyclic mechanical stretching induces autophagic cell death in tenofibroblasts through activation of PGE2 production. HPTFs grown in a custom-made silicone dish were cyclically stretched for $24 \mathrm{~h}$ followed by a 4 h rest. Non-stretched cells were used as the control. (A) The PGE2 concentration in the culture media after $4 \%, 8 \%$, or $12 \%$ stretching at $1 \mathrm{~Hz} .5 \leq n \leq 10,{ }^{*} \mathrm{P}<0.05$ vs. control. (B) The PGE2 concentration in the culture media after $12 \%$ stretching at $0.5,1.0$, or $1 \mathrm{~Hz} .5 \leq n \leq 10,{ }^{*} \mathrm{P}<0.05$ vs. control. (C - F) The PGE2 concentration in the culture media (C), the formation of AVOs and GFP-LC3-labeled vacuoles (D), the LC3-I, LC3II, and BECN1 protein expression (E), and cell death (F) after $12 \%$ stretching at $1.5 \mathrm{~Hz}$. When indomethacin $(25 \mu \mathrm{M})$ or celecoxib $(40 \mu \mathrm{M})$ was included in the treatment, cells were co-incubated with the compound during stretching. $5 \leq n \leq 10,{ }^{*} \mathrm{P}<0.05$.

autophagic cell death in tenofibroblasts, we speculated that repetitive mechanical loading could cause autophagic cell death in tenofibroblasts by stimulating endogenous PGE2 production. To test this hypothesis, we utilized apreviously reported in vitro stretching model $[4,20,21]$. HPTFs grown on a custom-made silicon dish were cyclically stretched for $24 \mathrm{~h}$ with $4 \%, 8 \%$ or $12 \%$ magnitude at a fixed frequency of $1 \mathrm{~Hz}$. After $4 \mathrm{~h}$ rest, the PGE2 concentration in the culture media was determined by ELISA. Although $4 \%$ stretching did not show significant effect, $8 \%$ and $12 \%$ stretching significantly increased PGE2 release in a magnitude-dependent manner (Fig. 4A). When cells were stretched at a fixed frequency of $0.5,1$, or $1.5 \mathrm{~Hz}$, PGE2 release significantly increased with the frequency of stretching (Fig. 4B). These results were consistent with previous reports [4]. The PGE2 concentration in the media reached about $7 \mathrm{ng} / \mathrm{ml}$ after $24 \mathrm{~h}$ stretching with $12 \%$ magnitude at a frequency 


\section{Cellular Physiology Cell Physiol Biochem 2015;36:24-33

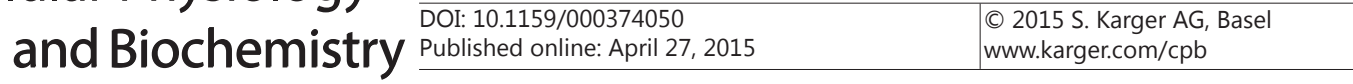 \\ Chen et al.: Cyclic Mechanical Stretching Triggers Autophagic Cell Death via PGE 2}

of $1.5 \mathrm{~Hz}$ (Fig. 4B). According to our studies with exogenous PGE2, this concentration (7 ng/ml) of PGE2 was sufficient to induce autophagic cell death in HPTFs. Indeed, we detected significantly higher percentage of cells with AVOs and GFP-LC3-labeled vacuoles (Fig 4D), elevated LC3-II and BECN1 levels (Fig. 4E), and increased cell death (Fig. 4F) in HPTFs mechanically stretched under these conditions. Blocking prostaglandin synthesis by COX inhibitor indomethacin or celecoxib significantly reduced stretching-induced PGE2 release (Fig. 4C), as well as autophagy and cell death (Fig. 4D - 4F). Taken together, these data demonstrate that cyclic mechanical stretching induces autophagic cell death in tenofibroblasts through activation of PGE2 production.

\section{Discussion}

In addition to its function as a metabolic recycling system to promote cellular homeostasis and survival, autophagy regulates vital processes such as programmed cell death, inflammation, and adaptive immune mechanisms [22]. Autophagy has been implicated in the pathogenesis of many diseases including cancer, neuro degenerative, cardiovascular, pulmonary, and autoimmune diseases [23, 24]. Although pharmacologic modulation of autophagy has shown some early promises, current therapeutic targeting of autophagy is limited by an incomplete understanding of how autophagy contributes to pathogenesis [23]. Targeting disease-specific autophagy pathways rather than general autophagy may be a more effective strategy for development of therapeutic agents.

Many studies suggested that sustained autophagy could lead to autophagic type II programmed cell death $[25,26]$. Autophagic cell death is morphologically defined as a type of cell death that occurs in the absence of chromatin condensation but is accompanied by largescale autophagic vacuolization of the cytoplasm [27]. In mammals, there is no clear evidence indicating the presence of such autophagic cell death under physiological settings. However, numerous reports showed the pro-death function of autophagy and situations where cell died by the form of " autophagic cell death," mostly from in vitro cell culture models [28]. In human cancer cells, there is a report showing a novel anti-cancer function of autophagic cell death in response to chemotherapy [29]. Such a finding is important as it provides evidence for possible exploration of inducing autophagic cell death for a therapeutic purpose in cancer.

Recently, excessive autophagic cell death has been detected in human tendinopathic tissues [16, 17], suggesting that autophagy plays an important role in the pathogenesis of tendinopathy. However, the factors causing autophagic cell death in tendinopathy are unclear. PGE2, a well-known inflammatory mediator of tendinopathy, has been reported to inhibit tenofibroblasts proliferation [6]. In the present study, we found that exposure to exogenous PGE2 induces cell death in cultured human tenofibroblasts via induction of autophagy. Incubation of HPTFs with exogenous PGE2 significantly increased cell death (Fig. 1), as well as the formation of autophagosomes and autolysosomes (assessed by electron microscopy, AO staining, and GFP-LC3 puncta) (Fig. 2A - 2C), a widely used marker for autophagy. PGE2treated cells also showed stimulated the conversion of LC3-I to LC3-II, and upregulated the expression of BECN1 (Fig. 2D). Blocking autophagy using inhibitors 3-methyladenine and chloroquine, or small interfering RNAs against autophagy genes Becn-1 and Atg-5 prevented PGE2-induced cell death (Fig. 3). These findings indicate that exogenous PGE2 causescell death in HPTFs through activation of autophagy. We subsequently demonstrated that repetitive mechanical loading stimulates PGE2 productionin HPTFs, which in turn induces autophagic cell death. Similar to previous findings [4], repetitive mechanical stretching at $8 \%$ and $12 \%$ magnitude for $24 \mathrm{~h}$ significantly increased PGE2 release by HPTFs in a magnitude-dependent manner. In addition, we observed increased cell death as well as activation of autophagy with 12\% stretching. Blocking PGE2 production using COX inhibitors indomethacin and celecoxib significantly reduced stretching-induced autophagy and cell death, indicating that PGE2 is the responsible factor for autophagic cell death. To our knowledge, this is the 


\section{Cellular Physiology Cell Physiol Biochem 2015;36:24-33 \begin{tabular}{l|l} 
DOI: 10.1159/000374050 & \\
and Biochemistry & 2015 S. Karger AG, Basel
\end{tabular}

first evidence linking PGE2 to autophagic cell death in tendinopathy. Although apoptotic cell death was not directly assessed in the present study, our data suggested that it also contributed to cell death induced by PGE2. Treatment of HPTFs with $15 \mathrm{ng} / \mathrm{ml} \mathrm{PGE2} \mathrm{for} 24$ h caused approximately $46 \%$ cell death; however, AVOs were detected in only about $16 \%$ of cells (Fig. 3B). Therefore, about 30\% cell death was possibly attributed to apoptosis, which is a well-recognized causative factor in tendinopathy. Further efforts are needed to elaborate the detailed death mechanisms involved in PGE2 activation.

The signaling pathways leading to autophagy are not fully understood; however, oxidative stress is among the main causative factors [30]. Repetitive mechanical stretching can activate oxidative stress-associated pathwaysin human tenofibroblasts [13]. The oxidative stressor $\mathrm{H}_{2} \mathrm{O}_{2}$ induces autophagic cell death in rotator cuff tenofibroblasts, and this is prevented by cyanidin, a strong antioxidant [31]. These findings suggest that oxidative stress-associated pathways are involved in the induction of autophagic cell death in tendinopathy, likely through cross talking with the IL-1 $\beta / C O X-2 /$ prostaglandin E2 pathway. Further studies are required to find out how these pathways crosstalk with each other to induce autophagic cell death in tendinopathy.

Currently, there are few good treatments for tendinopathy because of the lack of understanding of its pathogenesis. The new management strategies might target tendon stem cell proliferation and repair and restoration of matrix integrity. Advances in the research on autophagic cell death may help identify check points suitable for pharmaceutical intervention, and eventually help the development of effective therapy for tendinopathy.

\section{Disclosure Statement}

We declare that we have no conflicts of interest.

\section{Acknowledgments}

The study was supported by grants from Natural Science Foundation of China (No. 81372005).

\section{References}

1 Maffulli N, Longo UG, Loppini M, Spiezia F, Denaro V: New options in the management of tendinopathy. Open Access J Sports Med 2010;1:29-37.

- Koshima H, Kondo S, Mishima S, Choi HR, Shimpo H, Sakai T, Ishiguro N: Expression of interleukin-1beta, cyclooxygenase-2, and prostaglandin e2 in a rotator cuff tear in rabbits. J Orthop Res 2007;25:92-97.

-3 Almekinders LC, Baynes AJ, Bracey LW: An in vitro investigation into the effects of repetitive motion and nonsteroidal antiinflammatory medication on human tendon fibroblasts. Am J Sports Med 1995;23:119123.

- Wang JH, Jia F, Yang G, Yang S, Campbell BH, Stone D, Woo SL: Cyclic mechanical stretching of human tendon fibroblasts increases the production of prostaglandin e2 and levels of cyclooxygenase expression: $\mathrm{A}$ novel in vitro model study. Connect Tissue Res 2003;44:128-133.

-5 Devkota AC, Weinhold PS: Prostaglandin e(2), collagenase, and cell death responses depend on cyclical load magnitude in an explant model of tendinopathy. Connect Tissue Res 2010;51:306-313.

6 Cilli F, Khan M, Fu F, Wang JH: Prostaglandin e2 affects proliferation and collagen synthesis by human patellar tendon fibroblasts. Clin J Sport Med 2004;14:232-236.

7 Khan MH, Li Z, Wang JH: Repeated exposure of tendon to prostaglandin-e2 leads to localized tendon degeneration. Clin J Sport Med 2005;15:27-33.

8 Li H, Tang K, Deng Y, Xie M, Chang D, Tao X, Xu J: [effects of exogenous prostaglandin e2 on collagen content of achilles tendon of rabbits in vivo]. Zhongguo Xiu Fu Chong Jian Wai Ke Za Zhi 2012;26:352-358.

-9 Xu Y, Murrell GA: The basic science of tendinopathy. Clin Orthop Relat Res 2008;466:1528-1538. 


\section{Cellular Physiology Cell Physiol Biochem 2015;36:24-33

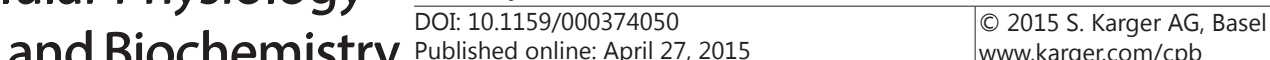 \\ Chen et al.: Cyclic Mechanical Stretching Triggers Autophagic Cell Death via $\mathrm{PGE}_{2}$}

10 Yuan J, Murrell GA, Wei AQ, Wang MX: Apoptosis in rotator cuff tendonopathy. J Orthop Res 2002;20:13721379.

11 Lian O, Scott A, Engebretsen L, Bahr R, Duronio V, Khan K: Excessive apoptosis in patellar tendinopathy in athletes. Am J Sports Med 2007;35:605-611.

-12 Scott A, Khan KM, Heer J, Cook JL, Lian O, Duronio V: High strain mechanical loading rapidly induces tendon apoptosis: An ex vivo rat tibialis anterior model. Br J Sports Med 2005;39:e25.

-13 Skutek M, van Griensven M, Zeichen J, Brauer N, Bosch U: Cyclic mechanical stretching of human patellar tendon fibroblasts: Activation of jnk and modulation of apoptosis. Knee Surg Sports Traumatol Arthrosc 2003;11:122-129.

14 Hale AN, Ledbetter DJ, Gawriluk TR, Rucker EB, 3rd: Autophagy: Regulation and role in development. Autophagy 2013;9:951-972.

15 Ryter SW, Mizumura K, Choi AM: The impact of autophagy on cell death modalities. Int J Cell Biol 2014;2014:502676.

16 Chen J, Wang A, Xu J, Zheng M: In chronic lateral epicondylitis, apoptosis and autophagic cell death occur in the extensor carpi radialis brevis tendon. J Shoulder Elbow Surg 2010;19:355-362.

17 Wu B, Chen J, Dela Rosa T, Yu Q, Wang A, Xu J, Zheng MH: Cellular response and extracellular matrix breakdown in rotator cuff tendon rupture. Arch Orthop Trauma Surg 2011;131:405-411.

18 Daido S, Kanzawa T, Yamamoto A, Takeuchi H, Kondo Y, Kondo S: Pivotal role of the cell death factor bnip3 in ceramide-induced autophagic cell death in malignant glioma cells. Cancer Res 2004;64:4286-4293.

19 Traganos F, Darzynkiewicz Z: Lysosomal proton pump activity: Supravital cell staining with acridine orange differentiates leukocyte subpopulations. Methods Cell Biol 1994;41:185-194.

$\longrightarrow 20$ Wang JH, Grood ES: The strain magnitude and contact guidance determine orientation response of fibroblasts to cyclic substrate strains. Connect Tissue Res 2000;41:29-36.

-21 Yang G, Im HJ, Wang JH: Repetitive mechanical stretching modulates il-1beta induced cox-2, mmp-1 expression, and pge2 production in human patellar tendon fibroblasts. Gene 2005;363:166-172.

22 Meijer AJ, Codogno P: Signalling and autophagy regulation in health, aging and disease. Mol Aspects Med 2006;27:411-425.

23 Choi AM, Ryter SW, Levine B: Autophagy in human health and disease. N Engl J Med 2013;368:651-662.

24 Zhou XJ, Zhang H: Autophagy in immunity: Implications in etiology of autoimmune/autoinflammatory diseases. Autophagy 2012;8:1286-1299.

25 Yu L, Alva A, Su H, Dutt P, Freundt E, Welsh S, Baehrecke EH, Lenardo MJ: Regulation of an atg7-beclin 1 program of autophagic cell death by caspase-8. Science 2004;304:1500-1502.

26 Gozuacik D, Kimchi A: Autophagy as a cell death and tumor suppressor mechanism. Oncogene 2004;23:2891-2906.

27 Kroemer G, Levine B: Autophagic cell death: The story of a misnomer. Nat Rev Mol Cell Biol 2008;9:10041010.

28 Shen HM, Codogno P: Autophagic cell death: Loch ness monster or endangered species? Autophagy 2010;7:457-465.

29 Xiong HY, Guo XL, Bu XX, Zhang SS, Ma NN, Song JR, Hu F, Tao SF, Sun K, Li R, Wu MC, Wei LX: Autophagic cell death induced by 5-fu in bax or puma deficient human colon cancer cell. Cancer Lett 2009;288:68-74.

30 Filomeni G, De Zio D, Cecconi F: Oxidative stress and autophagy: The clash between damage and metabolic needs. Cell Death Differ 2014;22:377-388.

-31 Kim RJ, Hah YS, Sung CM, Kang JR, Park HB: Do antioxidants inhibit oxidative-stress-induced autophagy of tenofibroblasts? J Orthop Res 2014;32:937-943. 